\title{
Influence of Patellar Tilt Angle in Merchant View on Postoperative Range of Motion in Posterior Cruciate Ligament-Substituting Fixed-Bearing Total Knee Arthroplasty
}

\author{
Jegyun Chon, MD, Taehyeon Jeon, MD, Jayeong Yoon, MD, Deukhee Jung, MD, \\ Chung-Han An, MD
}

Department of Orthopaedic Surgery, Daejeon Sun Hospital, Daejeon, Korea

\begin{abstract}
Background: We investigated whether the patellar tilt angle influences the maximum knee flexion after total knee arthroplasty (TKA) performed by using a posterior cruciate ligament-substituting (PS) fixed-bearing prosthesis in patients with relatively loose or tight flexion gap.

Methods: In this prospective cohort study, we followed up 169 patients for at least 2 years after TKA using PS fixed-bearing prosthesis. The patients were divided into two groups according to the flexion gap value-calculated by subtracting the thickness of the final bearing from the flexion gap measured intraoperatively under 200-cN.m force after patellar reduction and insertion of the final femoral and tibial components -into a relatively tight group (group T; 3-6.5 mm) and a relatively loose group (group L; 7-11 $\mathrm{mm})$. Patellar tilt angles and maximum non-weight-bearing active knee flexion angles were assessed postoperatively. Group $T$ was further divided into subgroup Tn if the patellar tilt angle was $<5^{\circ}$ and subgroup Tw if the angle was $\geq 5^{\circ}$. Pearson correlation test was used for the correlation analysis of the flexion gap, patellar tilt angle, and postoperative flexion range.

Results: The mean postoperative flexion was $137.3^{\circ}$ in group $T$ and $137.5^{\circ}$ in group $\mathrm{L}$. The mean patellar tilt angle was $6.5^{\circ}$ in group $T$ and $6.9^{\circ}$ in group L. In group $T$, a strong negative correlation $(r=-0.78, p<0.05)$ was observed between the patellar tilt and postoperative flexion range. However, further analysis revealed that only the subgroup Tw showed a strong negative correlation $(r=-0.76, p<0.05)$. Significant correlations were not found in the subgroup Tn and group L.

Conclusions: In TKA where a relatively tight flexion gap $(\leq 6.5 \mathrm{~mm})$ is created because of concerns about postoperative flexion instability due to a loose flexion gap, the patellar tilt angle should be $<5^{\circ}$ for maximal postoperative knee flexion.

Keywords: Arthroplasty knee, Flexion gap, Range of motion, Patellar tilt
\end{abstract}

Postoperative knee flexion is an important indicator of treatment success after total knee arthroplasty (TKA), a common procedure for osteoarthritis of the knee. ${ }^{1-4)}$ Knee

Received December 17, 2018; Accepted May 22, 2019

Correspondence to: Jegyun Chon, MD

Department of Orthopaedic Surgery, Daejeon Sun Hospital, 29 Mokjungro, Jung-gu, Daejeon 34811, Korea

Tel: +82-42-220-8860, Fax: +82-42-220-8464

E-mail: knee@naver.com range of motion, a clinical measure of knee function, has been reported to influence the ability to perform various daily activities and patient satisfaction. ${ }^{5-8)}$ In particular, deep flexion is important in the Asian population because prolonged squatting and sitting on the floor are often necessitated. ${ }^{9-12)}$ There are many factors that affect the range of motion after TKA. Flexion gap is one of such factors that influence postoperative flexion and instability of the knee in fixed-bearing TKA. ${ }^{13)}$ If the flexion gap is too tight, it will lead to a decreased postoperative flexion; if too 
loose, instability or dislocation. Although there are some reports on the correlation between the patellar tilt and postoperative knee flexion, ${ }^{14-16)}$ they have provided incon-

\begin{tabular}{|c|c|}
\hline Inclusion criteria & Exclusion criteria \\
\hline $\begin{array}{l}\text { - Osteoarthritis of the knee joint } \\
\text { - Body mass index }<25 \mathrm{~kg} / \mathrm{m}^{2} \\
\text { - Preoperative knee flexion } \geq 130^{\circ} \\
\text { - Mechanical varus knee angle } \\
\leq 20^{\circ}\end{array}$ & $\begin{array}{l}\text { - Body mass index } \geq 25 \mathrm{~kg} / \mathrm{m}^{2} \\
\text { - Preoperative knee flexion }<130^{\circ} \\
\text { - Mechanical varus knee angle } \\
>20^{\circ} \\
\text { - Mechanical valgus knee } \\
\text { - Rheumatoid arthritis of the knee } \\
\text { - Patellar resurfacing } \\
\text { - Instability or dislocation of } \\
\text { the knee joint after total knee } \\
\text { arthroplasty }\end{array}$ \\
\hline
\end{tabular}

Mechanical varus/valgus angle was measured by preoperative long bone anteroposterior view. sistent results, and they have not considered the influence of objective flexion gap. In this study, we measured the intraoperative flexion gap using a consistent force in TKA patients, then divided the patients into the relatively loose or tight flexion gap groups for comparison. The purpose of this study was to investigate whether the patellar tilt angle in the Merchant view will influence the postoperative maximum knee flexion in patients with a relatively loose or tight flexion gap measured in TKA performed using a posterior cruciate ligament-substituting (PS) fixed-bearing prosthesis. Our hypothesis was that the patellar tilt angle would affect postoperative range of motion, especially if the flexion gap were tight.

\section{METHODS}

After obtaining approval from the Institutional Review

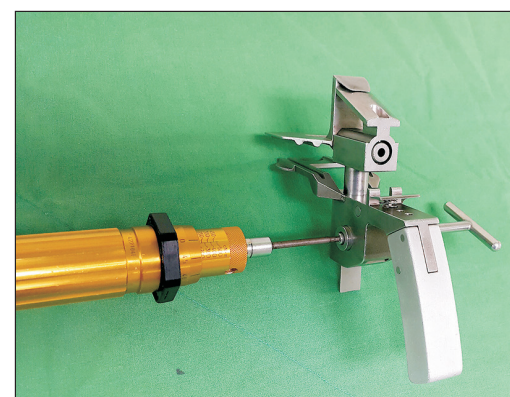

(A)
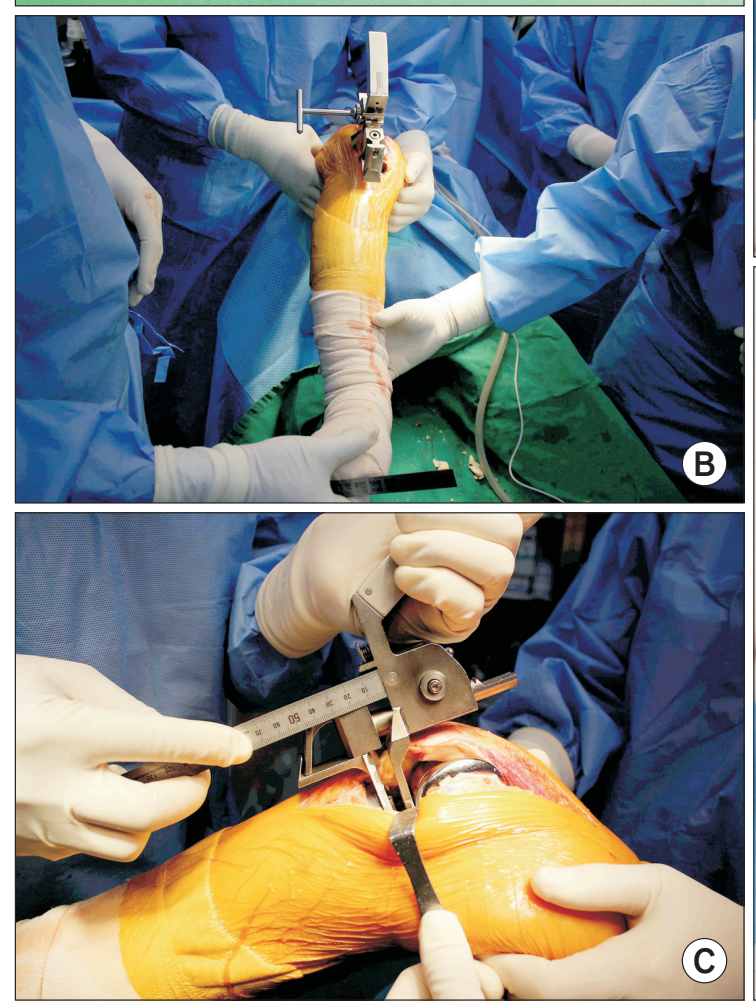

(C)

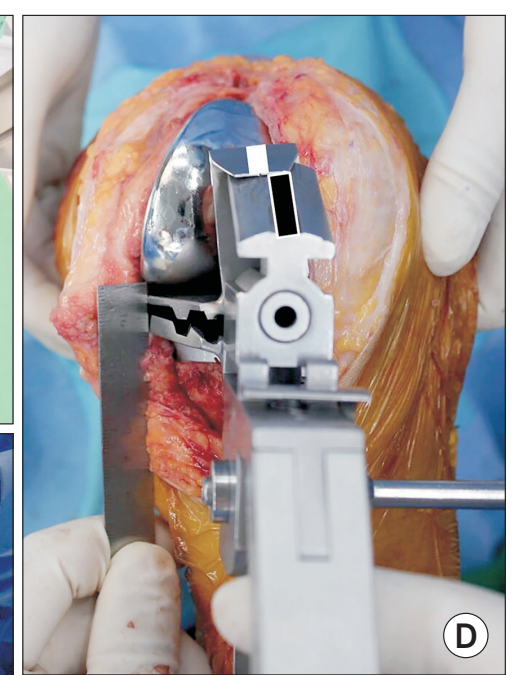

(D)

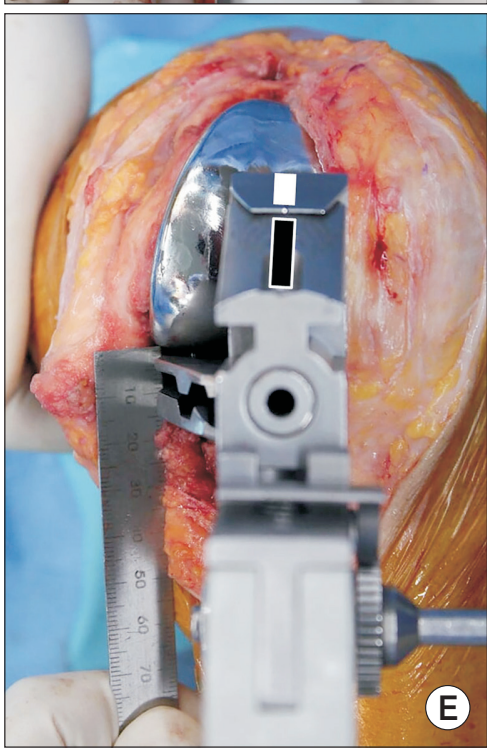

Fig. 1. Flexion gap measurement method. (A) The flexion gap was measured under $200 \mathrm{cN} \cdot \mathrm{m}$ force exerted by a tensor device and a torque driver. $(\mathrm{B}, \mathrm{C})$ The scale at the center of the tensor device balancer shows the balance between the medial and lateral gaps. We assembled a torque driver to the rack of the balancer and measured the gap distance across the two plates. (D) If the lateral gap is wider than the medial gap (unbalanced trapezoidal flexion gap), the white bar is not in parallel to the black bar due to the scissor effect on the tensor device. (E) However, when medial and lateral flexion gaps become equal after medial collateral ligament release, the white line is parallel to the black line on the tensor device in balanced quadrangular flexion gap. 
Board of Sun Medical Center (IRB No. DSH-18-07), we conducted a prospective cohort study involving 169 patients (153 women and 16 men) who were available for follow-up for at least 2 years after TKA using PS fixedbearing prosthesis (LOSPA; Corentec, Seoul, Korea). Informed consent was obtained from each patient before initiating the study. We established inclusion and exclusion criteria because various factors can affect the range of flexion after TKA, such as preoperative range of motion, body mass index (BMI), and preoperative tibiofemoral angle. ${ }^{16)}$

Patients were included if (1) they had degenerative osteoarthritis, (2) BMI was $<25 \mathrm{~kg} / \mathrm{m}^{2}$, (3) preoperative knee flexion was $\geq 130^{\circ}$, and (4) the mechanical axis was $\leq$ $20^{\circ}$ varus in preoperative long bone anteroposterior (AP) radiographs. Patients were excluded from the study if (1) their BMI was $\geq 25 \mathrm{~kg} / \mathrm{m}^{2}$, (2) preoperative knee flexion was $<130^{\circ}$, (3) preoperative mechanical knee axis was $>$ $20^{\circ}$ varus, (4) valgus mechanical axis was observed in the preoperative long bone AP view, (5) they had rheumatoid arthritis, (6) they underwent patellar resurfacing, or (7) they had instability or dislocation of the knee joint after TKA (Table 1).

In all cases, the flexion gap and postoperative knee range of motion were measured by the same surgeon (JC). Medial collateral ligament (MCL) releases were performed by using the pie crust technique to gain appropriate me-

\begin{tabular}{|c|c|c|}
\hline Variable & $\begin{array}{l}\text { Group T } \\
(n=50)\end{array}$ & $\begin{array}{l}\text { Group } L \\
(\mathrm{n}=119)\end{array}$ \\
\hline Postoperative flexion angle $\left({ }^{\circ}\right)$ & 137.3 (120 to 145$)$ & 137.5 (110 to 145$)$ \\
\hline Patellar tilt angle $\left(^{\circ}\right)$ & 6.5 (-2.5 to 22) & 6.9 (0.9 to 16.5$)$ \\
\hline
\end{tabular}

Values are presented as mean (range).

Group T: relatively tight group with flexion gap - bearing thickness $\leq 6.5$ $\mathrm{mm}$, Group L: relatively loose group with flexion gap - bearing thickness > $6.5 \mathrm{~mm}$.

\begin{tabular}{|c|c|c|}
\hline Variable & $\begin{array}{l}\text { Patellar tilt }<5^{\circ} \\
\quad(n=25)\end{array}$ & $\begin{array}{l}\text { Patellar tilt } \geq 5^{\circ} \\
\quad(n=25)\end{array}$ \\
\hline Postoperative flexion angle $\left({ }^{\circ}\right)$ & 141.6 (130 to 145$)$ & 133.0 (120 to 145$)$ \\
\hline Patellar tilt angle $\left({ }^{\circ}\right)$ & $3.0(-2.5$ to 4.7$)$ & 10.1 (5.1 to 22) \\
\hline
\end{tabular}

Values are presented as mean (range). chanical alignment and gap space. Lateral patellofemoral ligament and retinaculum releases were done for good patellofemoral tracking. Osteophytes were removed, and the inferior articular surface of the patella was flattened to prevent impingement between the patella and the bearing post during flexion. Patella resurfacing was not performed in all cases. Femoral bone cutting was performed before tibial bone cutting. Distal femoral cutting was performed at $6^{\circ}$ of valgus. In this setting, femoral external rotation was adjusted to make the balanced quadrangular flexion gap (equal medial and lateral gaps). For example, if a large medial gap is required for a balanced quadrangular flexion gap, the femoral external rotation was adjusted to $5^{\circ}$; if a small medial gap was required, the femoral external rotation was adjusted to $3^{\circ}$. Then, tibial bone cutting was performed with $0^{\circ}-3^{\circ}$ of tibial posterior slope, considering the flexion gap. Superficial MCL release was performed to transform the unbalanced trapezoidal gap (the lateral gap is wider than the medial gap) of the varus knee into a balanced quadrangular gap. As a result, the medial space of the flexion gap also became wider. Subsequently, with the femoral and tibial prostheses inserted and the patella reduced, the flexion gap was measured. Under 200-cN.m force exerted by a tensor device (Stryker Howmedica Osteonics, Allendale, NJ, USA) and a torque driver (Tohnichi torque driver; RTD500CN, Tohnichi, Tokyo, Japan), we flexed the knee to $90^{\circ}$ using a goniometer to create a rightangled flexion gap (Fig. 1). The flexion gap value was obtained by subtracting the thickness of the bearing from the measured flexion gap. After statistical analysis of the

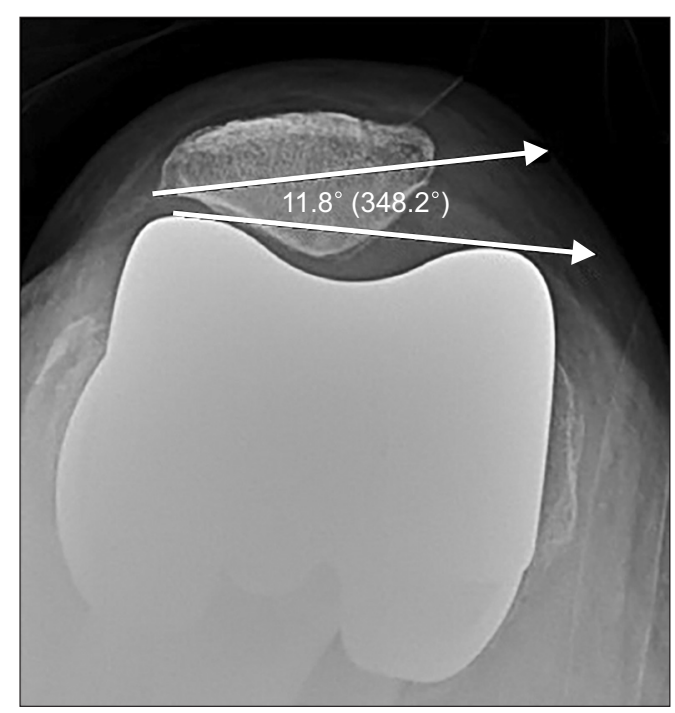

Fig. 2. Patellar tilt was measured as the angle between a line connecting the medial and lateral corners of the patella and another line connecting the anterior apex of medial and lateral femoral component condyles. 


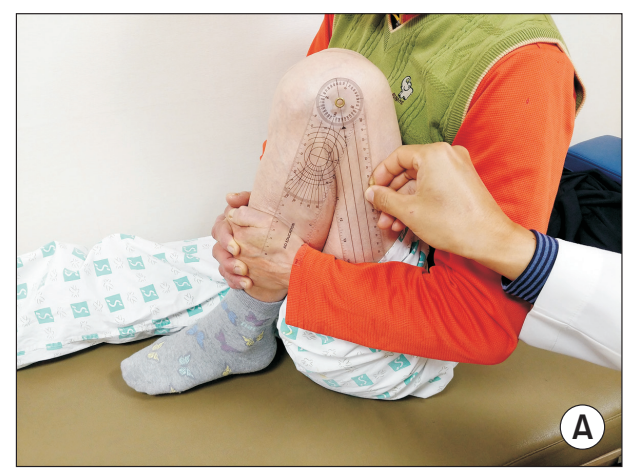

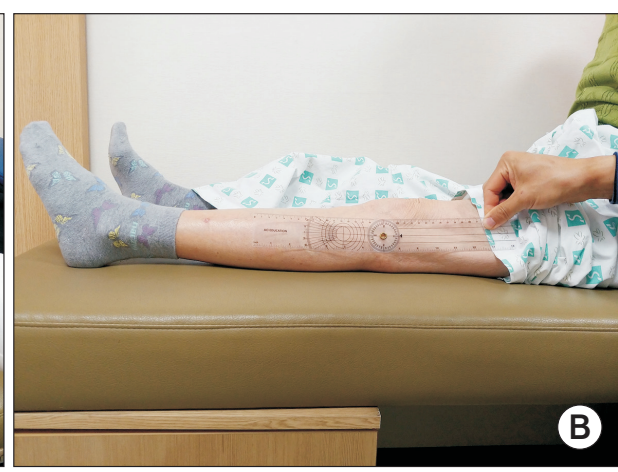

Fig. 3. Measurement of self-passive flexion (A) and extension (B) angles of the knee. Using a goniometer, we measured the intersection angle between midlines in the thigh and leg.

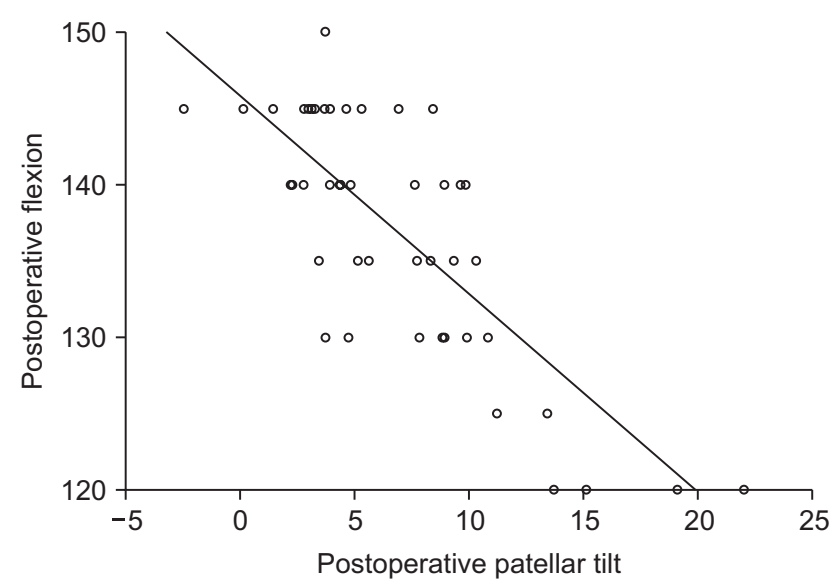

Fig. 4. Correlation between postoperative flexion and patellar tilt in Pearson correlation test in group $\mathrm{T}$ : a strong negative correlation $(r=$ $-0.78, p<0.05)$ was observed. Group T: the group with a relatively tight flexion gap (3-6.5 mm).

data, we found a negative correlation between the postoperative range of flexion and the flexion gap of $<6.5 \mathrm{~mm}$. So we divided the patients into the relatively tight flexion gap group (group $\mathrm{T}, \mathrm{n}=50$ ) where the flexion gap value was 3-6.5 $\mathrm{mm}$ and the relatively loose group (group L, $\mathrm{n}$ = 119) where the value was between 7 and $11 \mathrm{~mm}$ (Table 2). In group $T$, those with a patellar tilt angle of $<5^{\circ}$ were assigned to subgroup $\operatorname{Tn}(n=25)$ and those $\geq 5^{\circ}$ were assigned to subgroup $\mathrm{Tw}(\mathrm{n}=25)$ (Table 3$)$. All patients adhered to the standard rehabilitation protocol: progressive weight-bearing on the first day after surgery and continuous passive motion on the second day after surgery. During the minimum 2-year follow-up, patellar tilt angles were measured from Merchant views (Fig. 2); non-weightbearing self-passive flexion and extension of the knee were measured at the last follow-up by using a goniometer (Fig. 3). The IBM SPSS ver. 22.0 (IBM Corp., Armonk, NY, USA) was used for statistical analyses. The correlations between the patellar tilt and postoperative flexion were evaluated using the Pearson correlation test, with a $p$-value

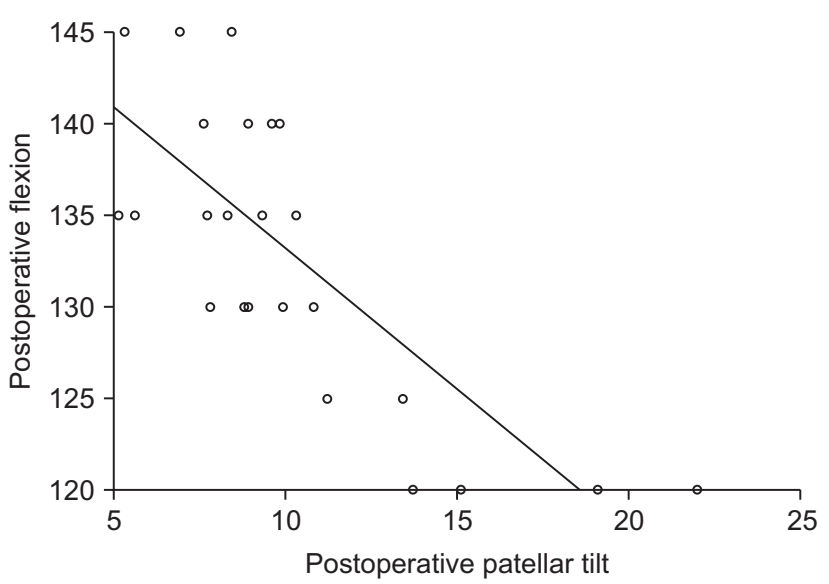

Fig. 5. Correlation between postoperative flexion and patellar tilt in Pearson correlation test in subgroup Tw. Of group T, the subgroup Tw with a patellar tilt $\geq 5^{\circ}$ showed a strong negative correlation $(r=-0.76, p$ $<0.05)$.

$<0.05$ considered significant.

\section{RESULTS}

The mean postoperative flexion angles were $137.3^{\circ}$ in group $\mathrm{T}$ and $137.5^{\circ}$ in group $\mathrm{L}$. The mean flexion gap value was $7.5 \mathrm{~mm}$. The mean patellar tilt angles in groups $\mathrm{T}$ and $\mathrm{L}$ are shown in Table 2 . In group $\mathrm{T}$, a strong negative correlation $(r=-0.78 ; p<0.05)$ was observed between the patellar tilt and postoperative flexion range (Fig. 4). However, on the subgroup analysis, the subgroup Tw (patellar tilt $\left.\geq 5^{\circ}\right)$ showed a strong negative correlation $(r=-0.76$; $p<0.05$ ) (Fig. 5), whereas the subgroup Tn (patellar tilt < $5^{\circ}$ ) did not show a significant negative correlation between the patellar tilt and the postoperative flexion range. In addition, no significant correlation was observed in group L. In other words, in cases with a relatively tight flexion gap $(\leq 6.5 \mathrm{~mm})$, the postoperative range of active knee flexion tended to decrease if the patellar tilt angle was $\geq 5^{\circ}$; however, if the patellar tilt angle was $<5^{\circ}$, regardless of flexion 
gap, patellar tilt was not correlated with postoperative range of active knee flexion.

\section{DISCUSSION}

The principal finding of this study is that patellar tilt affected the range of flexion after TKA only in the knee with a relatively tight flexion gap. Knee flexion is influenced by various factors, such as preoperative range of motion, component design, surgical technique, and rehabilitation. ${ }^{17)}$ Previous studies have reported on the correlation between patellar tilt and postoperative knee flexion in TKA. In a study by Kim et al., ${ }^{18)}$ when TKA was performed without a release of the lateral retinaculum, the mean patellar tilt was $6.12^{\circ}$, which is similar to $6.78^{\circ}$ obtained in our study despite release of the lateral retinaculum. Kawamura and Bourne ${ }^{16)}$ reported that the postoperative tilt angle of the patella may be a factor that affects the postoperative range of flexion. They suggested that the ideal postoperative tilt angle of the patella should be $<5^{\circ}$. Therefore, careful positioning of the patella and soft tissue balancing about the patella are advised to maximize flexion and improve patellar tracking after TKA. ${ }^{16,19)}$ In contrast, Dhollander et al. ${ }^{20)}$ reported that the patellar tilt did not seem to influence the postoperative flexion in high-flex design TKA.

However, these studies involved assessments of the patellar tilt angle and postoperative range of knee flexion without consideration of the flexion gap. Although many surgeons have recommended that the flexion gap should be 1 to $2 \mathrm{~mm}$ thicker than the bearing thickness for maximum knee flexion and stability after surgery, their recommendations are based on subjective observation. The objective flexion gap values may vary depending on the measurement method used by the surgeon. Accordingly, we investigated the correlations among three factors: the intraoperative flexion gap, patellar tilt angle, and postoperative maximum knee flexion angle. We found that in cases of TKA using a PS fixed-bearing prosthesis (LOSPA,
Corentec), if the goal is to achieve a relatively tight flexion gap of $\leq 6.5 \mathrm{~mm}$, the patellar tilt angle should be $<5^{\circ}$ to achieve the maximum active knee flexion angle. However, if the flexion gap is relatively loose ( $\geq 7 \mathrm{~mm}$ ), there is no correlation between the patellar tilt and maximum active knee flexion angle. Moreover, even when the surgeon intended to create a flexion gap that was $2 \mathrm{~mm}$ larger than the bearing thickness based on subjective feel, the final flexion gap measured by a more objective method using a tensor device and $200-\mathrm{cN} \cdot \mathrm{m}$ torque driver was at least $3 \mathrm{~mm}$ larger than the bearing thickness (mean, $7.5 \mathrm{~mm}$; range, $3-11 \mathrm{~mm}$ ). We think that this larger than the intended flexion gap was caused by measuring the gap with the thigh and foot raised at a right angle.

There are some limitations of the present study. First, the follow-up period of at least 2 years was relatively short. Miyagi et al. ${ }^{21)}$ demonstrated that the patellar tilt angle could increase after 10 years. Therefore, further studies with a longer follow-up are warranted to verify out results. Second, because most of the enrolled patients were women, the applicability of the study outcome may be limited in male patients. Third, the tibial slope was in the range of $0^{\circ}$ to $3^{\circ}$ in all knees to reduce its effect on the flexion gap; however, we did not consider the fact that the larger the slope, the greater the flexion gap after implantation. Finally, the patellar tilt angle in the Merchant view may be different from the patellar tilt observed in full flexion of the knee. So, further studies need to address additional methods for measuring patellar tilt angle. In conclusion, in TKA where a relatively tight flexion gap $(\leq 6.5 \mathrm{~mm})$ is created because of the concern about postoperative instability due to a loose flexion gap, the patellar tilt angle should be $<5^{\circ}$ for maximal postoperative knee flexion.

\section{CONFLICT OF INTEREST}

No potential conflict of interest relevant to this article was reported.

\section{REFERENCES}

1. Callahan CM, Drake BG, Heck DA, Dittus RS. Patient outcomes following tricompartmental total knee replacement: a meta-analysis. JAMA. 1994;271(17):1349-57.

2. Dieppe P, Basler HD, Chard J, et al. Knee replacement surgery for osteoarthritis: effectiveness, practice variations, indications and possible determinants of utilization. Rheumatology (Oxford). 1999;38(1):73-83.

3. Li PH, Wong YC, Wai YL. Knee flexion after total knee ar- throplasty. J Orthop Surg (Hong Kong). 2007;15(2):149-53.

4. Rorabeck CH, Murray P. The cost benefit of total knee arthroplasty. Orthopedics. 1996;19(9):777-9.

5. Anouchi YS, McShane M, Kelly F Jr, Elting J, Stiehl J. Range of motion in total knee replacement. Clin Orthop Relat Res. 1996;(331):87-92.

6. Dennis DA, Komistek RD, Stiehl JB, Walker SA, Dennis 
KN. Range of motion after total knee arthroplasty: the effect of implant design and weight-bearing conditions. J Arthroplasty. 1998;13(7):748-52.

7. Maloney WJ, Schurman DJ. The effects of implant design on range of motion after total knee arthroplasty: total condylar versus posterior stabilized total condylar designs. Clin Orthop Relat Res. 1992;(278):147-52.

8. Parsley BS, Engh GA, Dwyer KA. Preoperative flexion: does it influence postoperative flexion after posterior-cruciateretaining total knee arthroplasty? Clin Orthop Relat Res. 1992; (275):204-10.

9. Cho SH, Ha YC, Song HR, et al. High flex knee arthroplasty and range of motion. J Korean Orthop Assoc. 2004; 39(6):662-7.

10. Cho WS, Park JH, Kim JM, Hwang WY, Nam TS. Factors affecting range of motion after total knee arthroplasty. J Korean Orthop Assoc. 2003;38(7):683-8.

11. Jung WH, Ha YC, Lee JS. Range of motion after high-flexion posterior stabilized total knee arthroplasty: minimum 3-year follow up. J Korean Orthop Assoc. 2007;42(1):64-70.

12. Yamazaki J, Ishigami S, Nagashima M, Yoshino S. Hy-Flex II total knee system and range of motion. Arch Orthop Trauma Surg. 2002;122(3):156-60.

13. Higuchi H, Hatayama K, Shimizu M, Kobayashi A, Kobayashi T, Takagishi K. Relationship between joint gap difference and range of motion in total knee arthroplasty: a prospective randomised study between different platforms.
Int Orthop. 2009;33(4):997-1000.

14. Bengs BC, Scott RD. The effect of patellar thickness on intraoperative knee flexion and patellar tracking in total knee arthroplasty. J Arthroplasty. 2006;21(5):650-5.

15. Fukagawa S, Matsuda S, Mizu-uchi H, Miura H, Okazaki $\mathrm{K}$, Iwamoto Y. Changes in patellar alignment after total knee arthroplasty. Knee Surg Sports Traumatol Arthrosc. 2011;19(1):99-104.

16. Kawamura $\mathrm{H}$, Bourne RB. Factors affecting range of flexion after total knee arthroplasty. J Orthop Sci. 2001;6(3):248-52.

17. Kotani A, Yonekura A, Bourne RB. Factors influencing range of motion after contemporary total knee arthroplasty. J Arthroplasty. 2005;20(7):850-6.

18. Kim JH, Yoo BW, Kim CW. Influence of the rotational alignment of the femoral and patellar components on patellar tilt in total knee arthroplasty. Knee Surg Relat Res. 2015;27(3):163-7.

19. Kainz H, Reng W, Augat P, Wurm S. Influence of total knee arthroplasty on patellar kinematics and contact characteristics. Int Orthop. 2012;36(1):73-8.

20. Dhollander AA, Bassens D, Victor J, Verdonk P. Patellar tilt and thickness do not influence postoperative flexion in a high-flex design total knee arthroplasty. Knee Surg Sports Traumatol Arthrosc. 2013;21(12):2817-22.

21. Miyagi T, Matsuda S, Miura $\mathrm{H}$, et al. Changes in patellar tracking after total knee arthroplasty: 10-year follow-up of Miller-Galante I knees. Orthopedics. 2002;25(8):811-3. 